\title{
THE MULTIPARTITE RAMSEY NUMBER FOR THE 3-PATH OF LENGTH THREE
}

\author{
TOMASZ ŁUCZAK AND JOANNA POLCYN
}

\begin{abstract}
We study the Ramsey number for the 3-path of length three and $n$ colors and show that $R\left(P_{3}^{3} ; n\right) \leq \lambda_{0} n+7 \sqrt{n}$, for some explicit constant $\lambda_{0}=1.97466 \ldots$.
\end{abstract}

\section{INTRODUCTION}

Let $P_{3}^{3}$ be the 3-uniform hypergraph with the set of vertices $\{a, b, c$, $d, e, f, g\}$ and the set of edges $\{\{a, b, c\},\{c, d, e\},\{e, f, g\}\}$. The Ramsey number $R\left(P_{3}^{3} ; n\right)$ is the smallest integer $N$ such that any coloring of the edges of the complete 3 -uniform hypergraph $K_{N}^{3}$ on $N$ vertices with $n$ colors leads to a monochromatic copy of $P_{3}^{3}$. It is easy to see that $R\left(P_{3}^{3} ; n\right) \geq n+6$ (see [2, 4]) and it is believed that this lower bound is sharp, i.e. that $R\left(P_{3}^{3} ; n\right)=n+6$. However, so far this conjecture has been confirmed only for $n \leq 10$ (see [4, 6, 9, 10]). On the other hand, from the fact that for $N \geq 8$ each $P_{3}^{3}$-free 3-uniform hypergraph $H$ on $N$ vertices satisfies

$$
|H| \leq\left(\begin{array}{c}
N-1 \\
2
\end{array}\right)
$$

(see [1] and [5]), it follows that

$$
R\left(n ; P_{3}^{3}\right) \leq 3 n .
$$

In 7 the authors of this note improved the above upper bound to

$$
R\left(n ; P_{3}^{3}\right) \leq 2 n+\sqrt{18 n+1}+2 .
$$

Our argument relied on the fact that for 2-graphs the analogous multicolored Ramsey number for a 'usual' 2-path of length three is know to be $2 n+O(1)$, where the small hidden constant $O(1)$ depends only on divisibility of $n$ by 3 (see [3]). Thus, it seemed the method we used

2010 Mathematics Subject Classification. Primary: 05D10, secondary: 05C38, 05C55, 05C65.

Key words and phrases. Ramsey number, hypergraphs, paths.

The first author partially supported by NCN grant 2012/06/A/ST1/00261. 
in [7] could not be applied directly to get an upper bound better than $(\lambda+o(1)) n$, for $\lambda<2$.

The main result of this note is to match our previous approach with later results from [8] and get the following estimate for $R\left(P_{3}^{3} ; n\right)$.

Theorem 1. Let

$$
f(\gamma)=\left(\gamma^{3}-3 \gamma^{2}+6 \gamma-6\right)^{2}-72 \gamma(2-\gamma)(\gamma-1)^{2} .
$$

and let $\lambda_{0}=1.97466 \ldots$ be the solution to the equation $f(\gamma)=0$, such that $f(\gamma)>0$ whenever $\gamma \in\left(\lambda_{0}, 2\right)$. Then

$$
R\left(P_{3}^{3} ; n\right) \leq \lambda_{0} n+7 \sqrt{n} .
$$

\section{Proof of Theorem 1}

Our argument is based on the following decomposition lemma proved in [8]. Before we state it we need some definitions. We call a 3-graph $H$ quasi-bipartite if one can partition its set of vertices into three sets: $X=\left\{x_{1}, x_{2}, \ldots, x_{s}\right\}, Y=\left\{y_{1}, y_{2}, \ldots, y_{s}\right\}$, and $Z=\left\{z_{1}, z_{2}, \ldots, z_{t}\right\}$ in such a way that all the edges of $H$ can be written as $\left\{x_{i}, y_{i}, z_{j}\right\}$ for some $i=1,2, \ldots, s$, and $j=1,2, \ldots, t$. By a star with center $v$ we mean any 3 -graph in which each edge contains $v$. Then the following holds.

Lemma 2. For any $P_{3}^{3}$-free 3-graph $H$ there exists a partition of its set of vertices $V=V_{R} \cup V_{T} \cup V_{S}$, such that subhypergraphs of $H$ defined as $H_{R}=\left\{h \in H: h \cap V_{R} \neq \emptyset\right\}, H_{T}=H\left[V_{T}\right]$ and $H_{S}=H \backslash\left(H_{R} \cap H_{T}\right)=$ $\left\{h \in H\left[V \backslash V_{R}\right]: h \cap V_{S} \neq \emptyset\right\}$ satisfy the following three conditions:

(i) $\left|H_{R}\right| \leq 6\left|V_{R}\right|$,

(ii) $H_{T}$ is quasi-bipartite and $\left|H_{T}\right| \leq\left|V_{T}\right|^{2} / 8$,

(iii) $H_{S}$ is a family of disjoint stars such that centers of these stars are in $V_{T}$ whereas all other vertices are in $V_{S}$, and $\left|H_{S}\right| \leq\left(\begin{array}{c}\left|V_{S}\right| \\ 2\end{array}\right)$.

The following lemma is a direct consequence of the above result.

Lemma 3. Let $H$ be a $P_{3}^{3}$-free 3-graph $H$ on $N \geq 95$ such that for some $s,(N+3) / 2 \leq s \leq N-46$, we have

$$
|H| \geq\left(\begin{array}{c}
s-1 \\
2
\end{array}\right)+\left(\begin{array}{c}
N-s \\
2
\end{array}\right)
$$

and let $H=H_{R} \cup H_{T} \cup H_{S}$ be a decomposition of $H$ as described in Lemma 圆. Then $H_{S}$ contains a star on at least $s$ vertices. 
Proof. Let $V=V_{R} \cup V_{T} \cup V_{S}$ be a partition of the set of vertices $H$ given by Lemma 2. Note that $\left|V_{S}\right| \geq s-1$, since otherwise

$$
\begin{aligned}
|H| & \leq 6\left|V_{R}\right|+\frac{\left|V_{T}\right|^{2}}{8}+\left(\begin{array}{c}
\left|V_{S}\right| \\
2
\end{array}\right) \leq\left(\begin{array}{c}
\left|V_{S}\right| \\
2
\end{array}\right)+\frac{\left(N-\left|V_{S}\right|\right)^{2}}{8} \\
& \leq\left(\begin{array}{c}
s-2 \\
2
\end{array}\right)+\frac{(N-s+2)^{2}}{8}<\left(\begin{array}{c}
s-1 \\
2
\end{array}\right)+\left(\begin{array}{c}
N-s \\
2
\end{array}\right) .
\end{aligned}
$$

Recall that $H_{S}$ is a collection of disjoint stars. Suppose that the largest of these stars consists of at most $s-1>N / 2$ vertices. Then one can easily verify that the number of edges in $H_{S}$ is maximised if $H_{S}$ consists of two stars on $s-1$ and $\left|V_{S}\right|-(s-1)+2$ vertices respectively. Consequently

$$
\begin{aligned}
|H| & \leq 6\left|V_{R}\right|+\frac{\left|V_{T}\right|^{2}}{8}+\left(\begin{array}{c}
s-2 \\
2
\end{array}\right)+\left(\begin{array}{c}
\left|V_{S}\right|-s+2 \\
2
\end{array}\right) \\
& \leq\left(\begin{array}{c}
s-2 \\
2
\end{array}\right)+\left(\begin{array}{c}
N-s+1 \\
2
\end{array}\right)<\left(\begin{array}{c}
s-1 \\
2
\end{array}\right)+\left(\begin{array}{c}
N-s \\
2
\end{array}\right),
\end{aligned}
$$

again contradicting the fact that $|H| \geq\left(\begin{array}{c}s-1 \\ 2\end{array}\right)+\left(\begin{array}{c}N-s \\ 2\end{array}\right)$. Thus, $H_{S}$ contains a star on at least $s$ vertices.

Proof of Theorem 11. We show that if for given integers $N$ and $n$ one can find a coloring of edges of $K_{N}^{3}$ by $n$ colors without monochromatic copies of $P_{3}^{3}$, then $\gamma=(N-7 \sqrt{n}) / n<\lambda_{0}$ where $\lambda_{0}$ is defined in Theorem 1. Some parts of our argument are quite technical and, since we aim to prove the statement for every $n$, we start with few remarks which makes our future computations a bit easier.

Note that since $\lambda_{0}>1.97$, we may assume that $\gamma>1.9$. Moreover, due to (2), it is enough to consider $\gamma<2$. Finally, since $R\left(n ; P_{3}^{3}\right) \leq 3 n$ we can restrict to the case $n \geq 41$ (and hence $N>122$ ) because otherwise $3 n<1.9 n+7 \sqrt{n}$.

Thus, for $n \geq 41$ and $1.9<\gamma<2$, let us consider a coloring of edges of $K_{N}^{3}, N=\gamma n+7 \sqrt{n}$, by $n$ colors without monochromatic copies of $P_{3}^{3}$, and let $H_{i}$ denote the $P_{3}^{3}$-free hypergraph generated by the $i$-th color.

We say that the $i$ th color is rich if

$$
\left|H_{i}\right| \geq\left(\begin{array}{c}
n+6 \sqrt{n}-1 \\
2
\end{array}\right)+\left(\begin{array}{c}
N-n-6 \sqrt{n} \\
2
\end{array}\right) .
$$

Claim 4. At least $\beta$ n colors are rich, where

$$
\beta=\frac{\gamma^{3}-3 \gamma^{2}+6 \gamma-6}{6(\gamma-1)}
$$


Proof. Due to technical calculations it will be convenient to show the statement by contradiction. Denote the number of rich colors by $\beta n$ and assume that

$$
\beta<\frac{\gamma^{3}-3 \gamma^{2}+6 \gamma-6}{6(\gamma-1)}<\frac{1}{3}
$$

Since by (11), for each $i \in[n]$ we have $\left|H_{i}\right| \leq\left(\begin{array}{c}N-1 \\ 2\end{array}\right)$,

$$
\left(\begin{array}{c}
N \\
3
\end{array}\right)<\beta n\left(\begin{array}{c}
N-1 \\
2
\end{array}\right)+n(1-\beta)\left(\left(\begin{array}{c}
n+6 \sqrt{n}-1 \\
2
\end{array}\right)+\left(\begin{array}{c}
N-n-6 \sqrt{n} \\
2
\end{array}\right)\right) \text {. }
$$

Now substituting $N=\gamma n+7 \sqrt{n}$ and putting all leading terms on the left hand side of the equation we arrive at

$$
\begin{aligned}
{\left[\left(\gamma^{3}-3 \gamma^{2}+6 \gamma-6\right)\right.} & -\beta(6 \gamma-6)] n^{3}<\left[\beta(36 \gamma-30)-\left(21 \gamma^{2}-6 \gamma-30\right)\right] n^{5 / 2} \\
+ & {\left[\left(\beta(42-6 \gamma)-\left(150 \gamma-3 \gamma^{2}-105\right)\right] n^{2}\right.} \\
& -[6 \beta+400-42 \gamma] n^{3 / 2}-[2 \gamma-153] n-14 \sqrt{n} .
\end{aligned}
$$

But for $1.9<\gamma<2$ and $0 \leq \beta<1 / 3$ the right hand side of the above equation is smaller than $-19 n^{5 / 2}-157 n^{2}-316 n^{3 / 2}+150 n-14 \sqrt{n}$ which, in turn, is negative for all natural $n$. Consequently,

$$
\left[\left(\gamma^{3}-3 \gamma^{2}+6 \gamma-6\right)-\beta(6 \gamma-6)\right] n^{3}<0,
$$

and thus

$$
\beta>\frac{\gamma^{3}-3 \gamma^{2}+6 \gamma-6}{6(\gamma-1)}
$$

contradicting (41).

Recall that each $H_{i}$ is $P_{3}^{3}$-free and so one can apply to it Lemma 2 to get a decomposition of $H_{i}$ into three graphs, $H_{R}^{i} \cup H_{T}^{i} \cup H_{S}^{i}$. For all $i \in[n]$ let us 'uncolor' all the triples in $H_{R}^{i}$ and mark them 'blank', and set $\hat{H}_{i}=H_{T}^{i} \cup H_{S}^{i}$. Let $G_{i}$ be the graph whose edges are pairs which belong to at least two hyperedges of $\hat{H}_{i}$ and fewer than $6 \sqrt{n}$ blank triples. Note that, because of the structure of $\hat{H}_{i}, G_{i}$ is a forest consisting of stars.

We say that an edge of $G_{i}$ is private if it is not an edge of any other graph $G_{j}, j \neq i$, and public otherwise. By $e_{i}$ and $e_{i}^{\prime}$ we denote the number of private and public edges of $G_{i}$, respectively. The weight $w\left(G_{i}\right)$ of $G_{i}$ is defined as

$$
w\left(G_{i}\right)=e_{i}+e_{i}^{\prime} / 2 .
$$

Since $G_{i}$ is a forest we have also

$$
w\left(G_{i}\right) \leq e_{i}+e_{i}^{\prime}<N
$$


Note that at most

$\frac{3 \sum_{i=1}^{n}\left|H_{R}^{i}\right|}{6 \sqrt{n}} \leq \frac{3 \sum_{i=1}^{n} 6 N}{6 \sqrt{n}}=3 \sqrt{n} N=3 \sqrt{n}(\gamma n+7 \sqrt{n})<6 n^{3 / 2}+21 n$

pairs of $K_{N}^{2}$ belong to at least $6 \sqrt{n}$ blank triples. Since by the pigeonhole principle all pairs which are contained in fewer than $6 \sqrt{n}$ blank triples are edges of at least one $G_{i}$, we have

$$
\left(\begin{array}{c}
N \\
2
\end{array}\right)-6 n^{3 / 2}-21 n \leq \sum_{i \in[n]} w\left(G_{i}\right)
$$

Let us make the following easy yet crucial observation.

Claim 5. If a color $i$ is rich, then $G_{i}$ contains more than $2 w\left(G_{i}\right)-N$ private edges. All of them form a star.

Proof. Since $G_{i}$ is a forest we have $e_{i}+e_{i}^{\prime}<N$. Thus,

$$
w\left(G_{i}\right)=e_{i}+e_{i}^{\prime} / 2<e_{i}+N / 2-e_{i} / 2=\left(e_{i}+N\right) / 2,
$$

and so $G_{i}$ contains more than $2 w\left(G_{i}\right)-N$ private edges. Note also that, by Lemma 3, $H_{S}^{i}$ contains the unique largest star $F$ on at least $n+6 \sqrt{n}$ vertices. Let us denote the center of this star by $w$. Then each edge of $G_{i}$ which does not contain $w$ is clearly contained in fewer than $N-n-6 \sqrt{n}$ hyperedges of $\hat{H}_{i}$ and so belongs to at least $n$ triples of $\bigcup_{j \neq i} \hat{H}_{j}$. Thus, by the pigeonhole principle, each such edge must be public. Consequently, all private edges must contain $w$ and form in $G_{i}$ a large star.

Let $I$ denote the set of all rich colors. As an immediate corollary of Claim 5, we get the following inequality

$$
\left.\sum_{i \in I}\left(2 w\left(G_{i}\right)-N\right)<\sum_{i \in I} e_{i} \leq\left(\begin{array}{c}
|I| \\
2
\end{array}\right)+|I|(N-|I|)\right)
$$

which leads to

$$
\sum_{i \in I} w\left(G_{i}\right) \leq N|I|-|I|^{2} / 4-|I| / 4
$$


Thus, using (5) and (6) we get

$$
\begin{aligned}
\left(\begin{array}{c}
N \\
2
\end{array}\right)-6 n^{3 / 2}-21 n & \leq \sum_{i \in[n]} w\left(G_{i}\right)=\sum_{i \in I} w\left(G_{i}\right)+\sum_{i \notin I} w\left(G_{i}\right) \\
& <N|I|-|I|^{2} / 4-|I| / 4+\sum_{i \notin I} N \\
& =N|I|-|I|^{2} / 4-|I| / 4+(n-|I|) N \\
& \leq n N-|I|^{2} / 4 .
\end{aligned}
$$

Hence, using the estimate for the size of $I$ given by Claim 4 we arrive at

$$
\left(\frac{\gamma^{3}-3 \gamma^{2}+6 \gamma-6}{6(\gamma-1)}\right)^{2} \frac{n^{2}}{2} \leq \frac{|I|^{2}}{2}<2 n N-2\left(\begin{array}{c}
N \\
2
\end{array}\right)+12 n^{3 / 2}+42 n
$$

Now substituting $N=\gamma n+7 \sqrt{n}$ and putting all leading terms on the left hand side of the inequality results in the following formula

$$
\left(\frac{\left(\gamma^{3}-3 \gamma^{2}+6 \gamma-6\right)^{2}}{72(\gamma-1)^{2}}-\gamma(2-\gamma)\right) n^{2}<(26-14 \gamma) n^{3 / 2}+(\gamma-7) n+7 \sqrt{n}
$$

But for $1.9<\gamma<2$ and $n \geq 2$ we have

$$
(26-14 \gamma) n^{3 / 2}+(\gamma-7) n+7 \sqrt{n}<0
$$

and so

$$
\left(\frac{\left(\gamma^{3}-3 \gamma^{2}+6 \gamma-6\right)^{2}}{72(\gamma-1)^{2}}-\gamma(2-\gamma)\right) n^{2}<0
$$

Consequently,

$$
\left(\gamma^{3}-3 \gamma^{2}+6 \gamma-6\right)^{2}<72 \gamma(2-\gamma)(\gamma-1)^{2}
$$

which implies that $\gamma$ is smaller than $\lambda_{0}$ defined in Theorem 1 .

\section{REFERENCES}

[1] Z. Füredi, T. Jiang, R. Seiver, Exact solution of the hypergraph Turán problem for $k$-uniform linear paths, Combinatorica 34(3) (2014) 299-322.

[2] A. Gyárfás, G. Raeisi, The Ramsey number of loose triangles and quadrangles in hypergraphs, Electron. J. Combin. 19(2) (2012), \# R30.

[3] R.W. Irving, Generalised Ramsey numbers for small graphs, Discrete Math. 9 (1974) 251-264.

[4] E. Jackowska, The 3-color Ramsey number for a 3-uniform loose path of length 3, Australas. J. Combin. 63(2) (2015) 314-320.

[5] E. Jackowska, J. Polcyn, A. Ruciński, Turán numbers for 3-uniform linear paths of length 3, Electron. J. Combin. 23(2) (2016), \#P2.30

[6] E. Jackowska, J. Polcyn, A. Ruciński, Multicolor Ramsey numbers and restricted Turán numbers for the loose 3-uniform path of length three, arXiv:1506.03759v1. 
[7] T. Łuczak, J. Polcyn, On the multicolor Ramsey number for 3-paths of length three, Electron. J. Combin. 24(1) (2017), \#P1.27.

[8] T. Łuczak, J. Polcyn, Paths in hypergraphs: a rescaling phenomenon, arXiv:1706.08465.

[9] J. Polcyn, One more Turán number and Ramsey number for the loose 3-uniform path of length three, Discuss. Math. Graph Theory 37 (2017) 443-464.

[10] J. Polcyn, A. Ruciński, Refined Turán numbers and Ramsey numbers for the loose 3-uniform path of length three, Discrete Math. 340 (2017) 107-118.

Adam Mickiewicz University, Faculty of Mathematics and Computer Science, Umultowska 87, 61-614 Poznań, Poland

E-mail address: tomasz@amu.edu.pl

Adam Mickiewicz University, Faculty of Mathematics and Computer Science, Umultowska 87, 61-614 Poznań, Poland

E-mail address: joaska@amu.edu.pl 